\title{
Non-Thermal Radio Frequency and Static Magnetic Fields Increase Rate of Hemoglobin Deoxygenation in a Cell-Free Preparation
}

\author{
David Muehsam ${ }^{1,3 *}$, Parviz Lalezari ${ }^{2}$, Rukmani Lekhraj ${ }^{1}$, Provvidenza Abruzzo ${ }^{5}$, Alessandra Bolotta ${ }^{5}$, \\ Marina Marini ${ }^{5}$, Ferdinando Bersani ${ }^{6,7}$, Giorgio Aicardi ${ }^{3,7}$, Arthur Pilla ${ }^{4,8}$, Diana Casper ${ }^{1}$
}

1 Department of Neurosurgery, Albert Einstein College of Medicine and Montefiore Medical Center, Bronx, New York, United States of America, 2 Department of Medicine and Pathology, Albert Einstein College of Medicine and Montefiore Medical Center, Bronx, New York, United States of America, 3 Department of Human and General Physiology, University of Bologna, Bologna, Italy, 4 Department of Biomedical Engineering, Columbia University, New York, New York, United States of America, 5 Department of Histology, Embryology and Applied Biology, University of Bologna, Bologna, Italy, 6 Department of Physics, University of Bologna, Bologna, Italy, 7 Interdepartmental Center "L. Galvani", University of Bologna, Bologna, Italy, 8 Department of Orthopedics, Mount Sinai School of Medicine, New York, New York, United States of America

\begin{abstract}
The growing body of clinical and experimental data regarding electromagnetic field (EMF) bioeffects and their therapeutic applications has contributed to a better understanding of the underlying mechanisms of action. This study reports that two EMF modalities currently in clinical use, a pulse-modulated radiofrequency (PRF) signal, and a static magnetic field (SMF), applied independently, increased the rate of deoxygenation of human hemoglobin $(\mathrm{Hb})$ in a cell-free assay. Deoxygenation of $\mathrm{Hb}$ was initiated using the reducing agent dithiothreitol (DTT) in an assay that allowed the time for deoxygenation to be controlled (from several min to several hours) by adjusting the relative concentrations of DTT and $\mathrm{Hb}$. The time course of $\mathrm{Hb}$ deoxygenation was observed using visible light spectroscopy. Exposure for 10-30 min to either PRF or SMF increased the rate of deoxygenation occurring several min to several hours after the end of EMF exposure. The sensitivity and biochemical simplicity of the assay developed here suggest a new research tool that may help to further the understanding of basic biophysical EMF transduction mechanisms. If the results of this study were to be shown to occur at the cellular and tissue level, EMF-enhanced oxygen availability would be one of the mechanisms by which clinically relevant EMF-mediated enhancement of growth and repair processes could occur.
\end{abstract}

Citation: Muehsam D, Lalezari P, Lekhraj R, Abruzzo P, Bolotta A, et al. (2013) Non-Thermal Radio Frequency and Static Magnetic Fields Increase Rate of Hemoglobin Deoxygenation in a Cell-Free Preparation. PLoS ONE 8(4): e61752. doi:10.1371/journal.pone.0061752

Editor: Robert Lafrenie, Sudbury Regional Hospital, Canada

Received December 4, 2012; Accepted March 12, 2013; Published April 12, 2013

Copyright: (c) 2013 Muehsam et al. This is an open-access article distributed under the terms of the Creative Commons Attribution License, which permits unrestricted use, distribution, and reproduction in any medium, provided the original author and source are credited.

Funding: This work was supported in part by Ivivi Health Sciences, LLC, USA (www.ivivihealthsciences.com). No additional external funding was received for this study. The funders had no role in study design, data collection and analysis, decision to publish, or preparation of the manuscript.

Competing Interests: David Muehsam was a paid consultant for Ivivi Health Sciences, LLC, USA, for a portion of the duration of this study. Rukmani Lekhraj and Diana Casper received a portion of their salaries for other studies funded by Ivivi Health Sciences during the time of this study, but received no financial support for their contributions to this study. Arthur Pilla was a paid consultant for Ivivi Health Sciences LLC for the duration of this study. None of the other authors in this study received financial support or have other competing interests. This does not alter the authors' adherence to all the PLOS ONE policies on sharing data and materials.

*E-mail: davmumu@yahoo.com

\section{Introduction}

Identification of some of the mechanisms underlying EMF bioeffects and their clinical applications [1] has contributed to the development and more widespread use of more effective therapeutic signals [2]. It is clear that EMF modulation of nitric oxide signalling plays an important role in EMF therapeutics [26]. However, there may be other transduction pathways through which EMF signals could modulate tissue repair and growth. For example, several recent studies have reported EMF effects on human $\mathrm{Hb}$ in solution and erythrocyte suspensions, including decreases in viscosity [7] and changes in impedance [8], dielectric properties [9], dia- and para-magnetic properties [10], optical absorption [11] and in vivo deoxygenation [12]. In addition, radiofrequency mobile telephone signals have been shown to decrease $\mathrm{Hb}$ oxygen affinity in vitro [13] and changes in infrared absorption have been reported for low-frequency EMF [14]. Also, enhanced oxygen delivery by $\mathrm{Hb}$ is under investigation as a therapeutic strategy for the treatment of pathologies such as ischemia from stroke, cardiac disease and diabetic ulcers [15]. Thus, the present study aimed to characterize the effects on the rate of deoxygenation of human hemoglobin $(\mathrm{Hb})$ in an in vitro cellfree assay of a pulse-modulated radiofrequency (PRF) signal currently in clinical use for treatment of pain, edema and chronic wounds [1,2], and, applied independently, of a static magnetic field (SMF), from permanent ceramic magnets constructed for therapeutic applications and reported to reduce pain [1]. These non-thermal EMF modalities were chosen for this study due to their demonstrated efficacy in a variety of therapeutically relevant settings [1-6]. The reducing agent dithiothreitol (DTT) was employed for its ability to facilitate control of the time course of $\mathrm{Hb}$ deoxygenation by adjusting the ratio of DTT/Hb [16]. This study will show that exposure to PRF and SMF, yielded significant increases in the rate of $\mathrm{Hb}$ deoxygenation in the presence of the 
reducing agent DTT, observable several minutes to several hours after EMF exposure had ended.

\section{Materials and Methods}

\section{Hemoglobin Preparation}

Fresh human blood was obtained with written donor consent and approved for research by the Blood Bank at S.OrsolaMalpighi Hospital, Bologna according to the rules established by Legislative Decree 03-03-2005, article 9, paragraph 3, published in G.U. n. 85, 13.04.2005. Blood samples were also obtained from one author (DM) in accordance with New York State Consolidated Law, Public Health Article 24-A, Section 2442, without written approval of the Institutional Review Board of the Albert Einstein College of Medicine. Blood was drawn in EDTA anticoagulant tubes, centrifuged at $1000 \mathrm{x} \mathrm{g}$ for $10 \mathrm{~min}$ and plasma removed. The packed erythrocytes were then washed in $0.85 \%$ sodium chloride solution and centrifuged at $1000 \mathrm{x} \mathrm{g}$ for $10 \mathrm{~min}, 3$ times. The packed, washed erythrocytes were then hemolyzed using distilled water, the resulting solution centrifuged at $1000 \mathrm{x} \mathrm{g}$ for $10 \mathrm{~min}$, and the Hb-containing supernatant recovered using a Pasteur pipette. Red cell ghosts were sedimented by additional centrifugation, and the resulting solution containing 2-2.5 $\mathrm{mM}$ oxyHb (measured by visible light spectroscopy [17]), was stored in $1.5 \mathrm{~mL}$ aliquots at $-80^{\circ} \mathrm{C}$ until used for each experiment. Ten $\mathrm{mL}$ reaction mixtures were prepared using 100 $120 \mu \mathrm{M} \mathrm{Hb}$ in $50 \mathrm{mM}$ Hepes buffer (pH 7.2, Sigma-Aldrich, USA), and deoxygenation initiated with $20 \mathrm{mM}$ DTT at $22 \pm 0.1^{\circ} \mathrm{C}$. $\mathrm{pH}$ was checked with a digital $\mathrm{pH}$ meter (Fisher AB15 BioBasic, USA) and carefully maintained at 7.2 for all hemoglobin samples used in this experiment. Immediately upon addition of DTT, the $10 \mathrm{~mL}$ reaction mixture volume was divided into $1 \mathrm{~mL}$ aliquots in sealed $1.5 \mathrm{~mL}$ microfuge tubes. No attempt was made to alter the gaseous environment within the tubes.

\section{EMF Exposure}

All hemoglobin samples were exposed to the ambient magnetic field, which was measured using a digital Gauss/Tesla meter (model 7010, F.W. Bell, USA) to be $40.5 \pm 2 \mu \mathrm{T}, 59$ degrees from horizontal (vertical component $=34.7 \pm 2 \mu \mathrm{T}$, horizontal component $=21.0 \pm 2 \mu \mathrm{T})$. The PRF signal is approved by the US FDA for post-operative pain and edema. The signal consisted of a 27.12 MHz sinusoidal carrier (derived from the carrier frequency reserved and cleared worldwide for short wave diathermy) configured to operate nonthermally through pulse modulation in $4 \mathrm{~ms}$ bursts, repeating at $5 \mathrm{~Hz}$ and peak magnetic field amplitude of $10 \pm 1 \mu \mathrm{T}$ (Roma3, Ivivi Health Sciences, San Francisco, CA, USA). These pulse modulation parameters were chosen on the bases of theoretical modelling and published reports of bioeffects at the cellular level, and healing at the animal and clinical levels [1-3,18-24]. The PRF signal was delivered with a $20 \mathrm{~cm}$ circular single-turn antenna (coil) oriented vertically, creating a $10 \times 10 \times 5 \mathrm{~cm}$ region of field homogeneity in the central area of the plane of the coil within which a plastic carrier held five upright $1.5 \mathrm{~mL}$ microfuge tubes, each containing $1 \mathrm{~mL}$ reaction volume. Each $\mathrm{Hb}$ sample was contained in a cylindrical volume of $2.5 \mathrm{~cm}$ in height and $0.8 \mathrm{~cm}$ diameter. For this target size, using Faraday's Law of Induction, the mean peak induced electric field is $3 \pm 1 \mathrm{~V} /$ m. PRF field parameters were assessed and verified for each experiment using a National Institute of Standards and Testing traceable electrostatically shielded loop probe $1 \mathrm{~cm}$ in diameter (model 100A, Beehive Electronics, Sebastopol, CA, USA) connected to a calibrated 100-MHz oscilloscope (model 2012B, Tektronix, Beaverton, OR, USA). The output of the loop probe was calibrated at $27.12 \mathrm{MHz}$ by measurement of output power using a spectrum analyser (model 8567A, Hewlett Packard, New York, NY, USA) and the probe calibration factor for conversion to magnetic field amplitude at $27.12 \mathrm{MHz}$ was certified and given by the manufacturer. SMF exposure was delivered using circular permanent ceramic magnets of $3.8 \mathrm{~cm}$ diameter and $1.3 \mathrm{~cm}$ thickness, constructed for therapeutic applications (Magnetherapy, West Palm Beach, FL, USA), and composed of compacted and sintered strontium ferrite $\left(\mathrm{SrO}-6\left(\mathrm{Fe}_{2} \mathrm{O}_{3}\right)\right)$, encased in plastic. These magnets are axially magnetized to have a single north $(\mathbf{N})$ and a single south (S) pole on each circular face, and manufactured to produce highly uniform field strength across each face. For all experiments, magnets were arranged with circular faces oriented vertically with $1.1 \mathrm{~cm}$ gaps between the parallel surfaces. In order to simultaneously expose five $1.5 \mathrm{~mL}$ microfuge tubes, two $1.1 \mathrm{~cm}$-wide treatment regions were formed using a central magnet flanked by pairs of magnets on either side. This (NS)(NS)-gap-(NS)-gap-(NS)(NS) configuration produced two cylindrical treatment regions of $1.1 \mathrm{~cm}$ width and $3.3 \mathrm{~cm}$ in diameter with uniform magnetic field (to $\pm 3 \%$ ), in which the $\mathrm{Hb}$ samples were exposed. Within each treatment region the horizontal component of the magnetic field (perpendicular to the magnet surface) was $186 \pm 6 \mathrm{mT}$, a field strength similar to those commonly employed in therapeutic applications [25]. The maximal value of the horizontal and vertical components (both parallel to the magnet surface) was $4.0 \pm 0.6 \mathrm{mT}$. SMF components were measured using a digital magnetometer (Model 450, gaussmeter with MMT-6J02-VG transverse Hall effect probe with $1 \mathrm{~mm}$ resolution, Lake Shore Cryotronics, Westerville, OH, USA). For each experiment, five $1.5 \mathrm{~mL}$ microfuge tubes containing the hemoglobin preparation were exposed to PRF or SMF for 10$30 \mathrm{~min}$, and 5 tubes were simultaneously exposed to ambient geomagnetic conditions (control) on the laboratory bench, approximately 3 meters away from exposed samples. At this distance contributions from either PRF or SMF to the control condition were undetectable. Temperature variations between exposed and control samples were less than $\pm 0.1^{\circ} \mathrm{C}$ [Fisher AB15 BioBasic, Waltham, MA, USA].

\section{Spectrophotometric Analysis}

After control and EMF exposure, triplicate $300 \mu \mathrm{L}$ samples were taken from each $1.5 \mathrm{~mL}$ microfuge sample tube, pipetted into an open 96-well flat-bottom plate (Fisher Scientific, USA) and the concentrations of oxy, deoxy and metHb were measured spectrophotometrically (SpectraMax 190, Molecular Devices, Sunnyvale, CA, USA). The plate remained in the spectrophotometer at $22^{\circ} \mathrm{C}$ during the reaction, and the time course of deoxygenation was determined using the method of Benesch et al. [17], which employs a weighted linear combination of optical densities at 560, 576 and $630 \mathrm{~nm}$ to determine oxyHb, deoxyHb and metHb concentrations. Stock Hb solutions (in Hepes, pH 7.2) and spectra from Hb/DTT data at $\mathrm{t}=0$ (i.e. after $30 \mathrm{~min}$ EMF/ Control exposures) were assayed for oxy, deoxy and metHb at 560, 576 and $630 \mathrm{~nm}$ [17]. The mean ratio of deoxy/oxy for $\mathrm{Hb}$ stock solutions was $3.9 \% \pm 0.8 \%$. For the Hb/DTT data at $\mathrm{t}=0$, the mean deox/oxy was $3.3 \% \pm 0.6 \%$. The two datasets do not differ significantly $(\mathrm{P}=0.44, \mathrm{n}=5)$. The mean ratio of metHb/ oxyHb was $21.8 \% \pm 3.8 \%$ for stock solutions and $25.6 \% \pm 3.7 \%$ for $\mathrm{Hb} / \mathrm{DTT}$ data at $\mathrm{t}=0$, indicating no significant differences $(\mathrm{P}=0.33, \mathrm{n}=5)$. Optical densities at 540, 560, 576 and $630 \mathrm{~nm}$ were measured immediately after EMF exposure and at successive 1 to 30 min intervals, until maximal deoxygenation was observed. Calibration studies confirmed that the kinetics of deoxygenation could be controlled by adjusting the ratio of $\mathrm{DTT} / \mathrm{Hb}$ concen- 
trations [16]. Under the conditions stated above, deoxygenation occurred between 140 and $160 \mathrm{~min}$ after EMF exposure. Deoxygenation time was reduced by the addition of $5 \mathrm{M}$ of urea to between $60-85 \mathrm{~min}$ after EMF exposure. In the absence of DTT, no significant deoxygenation of $\mathrm{Hb}$ occurred up to 3 hours (data not shown). Changes in oxyHB concentration with time are summarized in the figures, and maximal differences between deoxy and metHb for EMF exposures vs controls are reported in the text. All figures and data shown are the results of single experiments that have been repeated 2-10 times.

\section{Statistical Analysis}

Results were compared using the Student's t test, or one-way repeated measures ANOVA with Holm-Sidak post hoc analysis, and Fieller's method for the variance of ratios, as required (Sigmastat 3.0, Systat, Chicago, IL) and are reported as means \pm SEM. Significance was accepted at $\mathrm{P} \leq 0.05$.

\section{Results}

Immediately after $30 \mathrm{~min}$ of PRF or SMF exposure, no differences in $\mathrm{Hb}$ visible light spectra between PRF, SMF and control (ambient magnetic field only) samples were detected. However, changes in visible light spectra were observed in the $540-630 \mathrm{~nm}$ region during the time of most rapid deoxygenation, which occurred in this experiment at 140-160 min after EMF exposure. Figure 1 illustrates typical visible light spectra for PRF, SMF and control exposure conditions, drawn from a single exposure tube for each EMF condition. Deoxygenation occurred at an earlier time following PRF and SMF exposures (traces with one peak), as compared to control samples exposed only to the ambient geomagnetic laboratory environment (trace with two peaks). In trials employing PRF exposure $(n=5$ tubes for each EMF exposure condition) using $20 \mathrm{mM}$ DTT, $150 \mathrm{~min}$ after a single $30 \mathrm{~min}$ exposure, PRF-treated samples exhibited a $30.8 \pm 10.3 \%$ reduction in oxyHb as compared to controls $(14.6 \pm 1.3 \mu \mathrm{M}$ vs. $21.1 \pm 2.5 \mu \mathrm{M}, \mathrm{P}<0.03$ ) (Figure 2). Concomitant, significant increases in deoxy $\mathrm{Hb}(86.9 \pm 1.1 \mu \mathrm{M}$ vs. $82.8 \pm 1.8 \mu \mathrm{M}, \quad \mathrm{P}<0.05)$ and $\mathrm{metHb} \quad(42.2 \pm 1.4 \mu \mathrm{M}$ vs. $37.1 \pm 1.4 \mu \mathrm{M}, \mathrm{P}<0.02)$ also occurred. For the ratio of DTT/ oxyHb concentrations employed in these trials $(20 \mathrm{mM}$ DTT; $120 \mu \mathrm{M}$ oxyHb), $150 \mathrm{~min}$ corresponded to the time of most rapid deoxygenation. Addition of $5 \mathrm{M}$ urea to the reagent solution decreased the time to required deoxygenate to approximately 6575 min after reaction initiation. For these conditions, the maximal difference between PRF-treated and control samples occurred at 73 min, with PRF-treated samples showing a $70.5 \pm 9.7 \%$ reduction in oxyHb as compared to controls $(8.5 \pm 2.1 \mu \mathrm{M}$ vs. $28.8 \pm 6.3 \mu \mathrm{M}, \mathrm{P}<0.03$ ) (Figure 3). Concomitantly, there were significant increases in deoxy $\mathrm{Hb}(54.4 \pm 2.2 \mu \mathrm{M}$ vs. $40.9 \pm 5.2 \mu \mathrm{M}$, $\mathrm{P}<0.04)$ and metHb $(73.4 \pm 1.4 \mu \mathrm{M}$ vs. $61.0 \pm 2.3 \mu \mathrm{M}, \mathrm{P}<0.02)$. Under similar conditions (30 mM DTT, $5 \mathrm{M}$ urea, $\mathrm{n}=5$ ) SMF treated samples (10-30 min exposure) underwent nearly complete deoxygenation before the untreated (control) samples began to deoxygenate $(\mathrm{P}<0.002$ for $42 \mathrm{~min}<\mathrm{t}<58 \mathrm{~min})$. For these $\mathrm{SMF}$ treated samples, the most rapid deoxygenation occurred approximately $10 \mathrm{~min}$ earlier than for control samples (Figure 4). Under these conditions, SMF-treated samples substantially completed DTT-induced deoxygenation before untreated samples began to lose $\mathrm{O}_{2}$, an effect that was visibly observable in the 96-well spectrophotometer plate, as shown in the photo in Figure 5, taken approximately $46 \mathrm{~min}$ after the reaction was initiated. Experiments were also carried out for which: 1) Hb was treated with PRF or SMF for $30 \mathrm{~min}$ prior to introduction into the deoxygenation solution (20 mM DTT+5M urea in $50 \mathrm{mM}$ Hepes) and 2) the deoxygenation solution was treated with PRF or SMF for $30 \mathrm{~min}$ prior to introduction of $\mathrm{Hb}$. In both cases the difference between PRF- or SMF-treated and control samples was not significant. Also, 10 min and $30 \mathrm{~min}$ PRF and SMF treatment durations were compared (in $20 \mathrm{mM}$ DTT $+5 \mathrm{M}$ urea in $50 \mathrm{mM}$ Hepes), with no significant differences observed in the time course of deoxygenation. In the absence of DTT, no PRF or SMF effects were observed on the $\mathrm{Hb}$ oxidation state. The SMF sensitivity of this $\mathrm{Hb}$ deoxygenation assay was repeated at the University of Bologna, Italy. Initial experiments were performed in New York with $\mathrm{Hb}$ from a single donor, and subsequent trials in Bologna used $\mathrm{Hb}$ from 3 different donors, with similar results.

\section{Discussion and Conclusions}

The results from this study indicate that exposure to either PRF or SMF, applied independently, can alter the rate of DTT-induced deoxygenation of human $\mathrm{Hb}$ in an in vitro cell-free preparation, resulting in more rapid $\mathrm{Hb}$ deoxygenation. To the authors' knowledge, this is the first report showing that PRF and SMF produced similar effects in the same biological system, in the same study. The rate of $\mathrm{Hb}$ deoxygenation is non-linear in time and is dependent upon the ratio of $\mathrm{DTT} / \mathrm{HbO}_{2}$. For this assay, the use of DTT as a reducing agent is preferable to other reducing agents, due to the ease with which the time to deoxygenation can be controlled [16]. It is interesting to note that a similar EMF sensitivity, for $\mathrm{Hb}$ exposed to a GSM mobile phone signal, was observed using sodium dithionite [13]. This suggests that EMF acted upon a functional aspect of the $\mathrm{Hb}$ deoxygenation process itself, and that this effect was not specific to the reducing agent employed in this study. The observation that EMF pre-treatment of $\mathrm{Hb}$ alone, or of the deoxygenation solution itself, failed to yield a significant effect suggests that both PRF and SMF exposures acted upon the interaction of $\mathrm{Hb}$ with the deoxygenating solution. The addition of $5 \mathrm{M}$ urea reduced the time to deoxygenate and rendered both the PRF and SMF effects more easily observable. Urea creates an extended cloud of waters that only weakly participates in the hydrogen bonding network of bulk water, facilitating the loosening of the protein structure [26] and destabilizing the water-oxyHb structures that act as key allosteric mediators of the $\mathrm{Hb} \mathrm{T}-\mathrm{R}$ transition [27], thus reducing the energy required to deoxygenate. However, it is unclear whether the addition of urea affected the primary PRF or SMF transduction or merely renders the EMF effects more easily observable. No differences were observed between 10 and 30 min SMF and PRF treatment durations. Effects were observable several min to several hours after PRF or SMF exposure was removed, suggesting that these EMF modalities modified protein/solvation structure in a manner that altered the energy required for deoxygenation.

This study does not provide sufficient detail to allow for the elucidation of the mechanisms of action of EMFs on hemoglobin, or to determine if SMF and PRF act upon the same submolecular targets to produce the effects observed here. Also, further studies are required to determine the range of field parameters for which this assay exhibits sensitivity. However, the sensitivity and biochemical simplicity of the assay developed here suggests its usefulness for future studies that may further establish basic biophysical EMF transduction mechanisms. The PRF signal was developed using a theoretical model for the effect of the induced electric field on ion binding kinetics at protein aqueous interfaces $[1-3,18]$. It should be noted that PRF transduction through the induced electric field is distinct from the transduction pathway involved in SMF effects, for which no electric field is present. This 


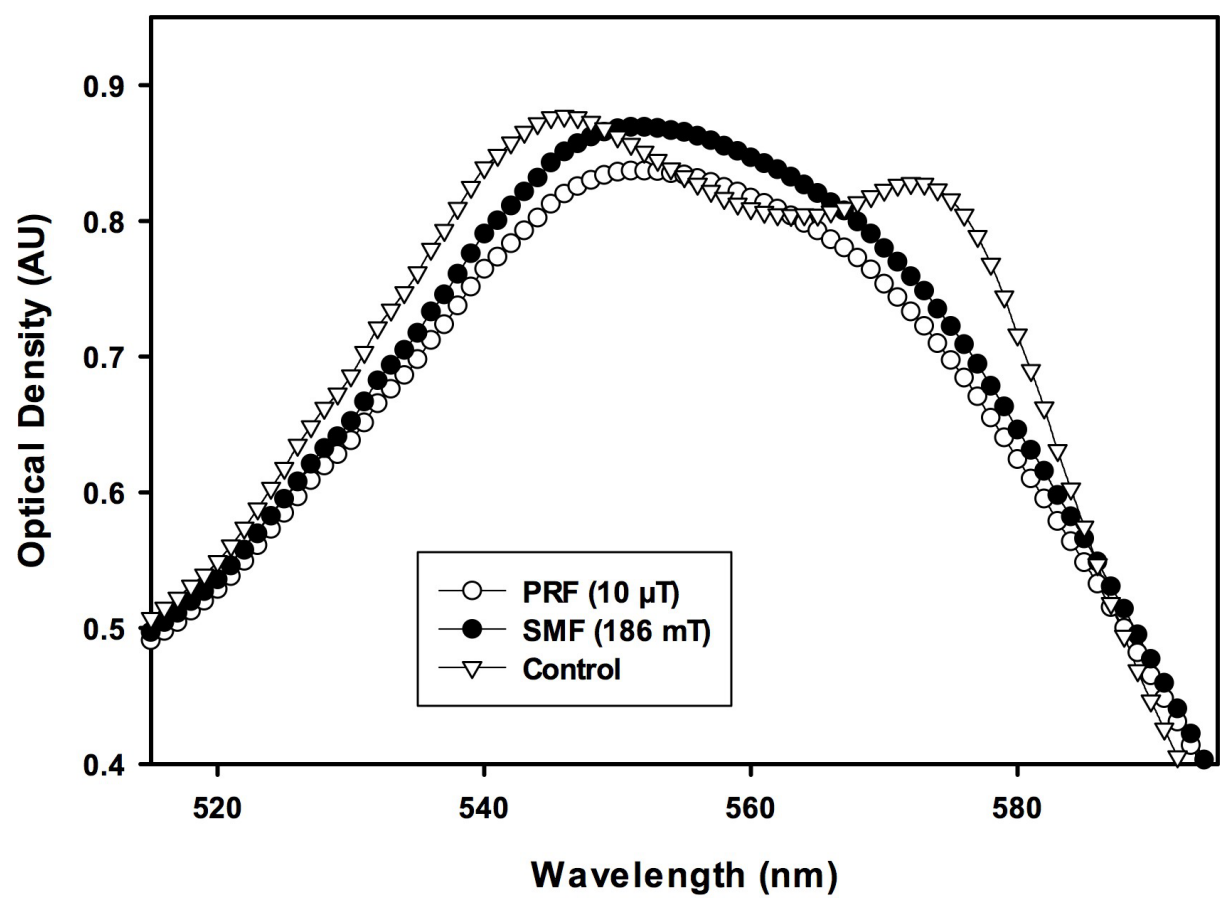

Figure 1. EMF effect on hemoglobin $(\mathbf{H b})$ visible light spectra. Spectra representative of the effects of pulsed radiofrequency (PRF) signal and $186 \mathrm{mT}$ static magnetic field (SMF) on $\mathrm{Hb}$ visible light spectra during bHb)deoxygenation. Data shown are typical of samples drawn from a single tube for each EMF exposure condition, shown here at $150 \mathrm{~min}$ after a single $30 \mathrm{~min}$ EMF exposure. Deoxygenation of $100 \mu \mathrm{M} \mathrm{Hb}$ was carried out in $50 \mathrm{mM}$ Hepes buffer ( $\mathrm{pH} 7.2)$ using the reducing agent dithiothreitol $(20 \mathrm{mM})$ at $22^{\circ} \mathrm{C}$, and is characterized here by the passage of the spectrum from a two-peaked to one-peaked form. Deoxygenation occured at an earlier time for EMF exposed samples (traces with one peak), as compared to control samples exposed only to the ambient geomagnetic laboratory environment (trace with two peaks). The EMF effect was observable at the time of most rapid deoxygenation.

doi:10.1371/journal.pone.0061752.g001

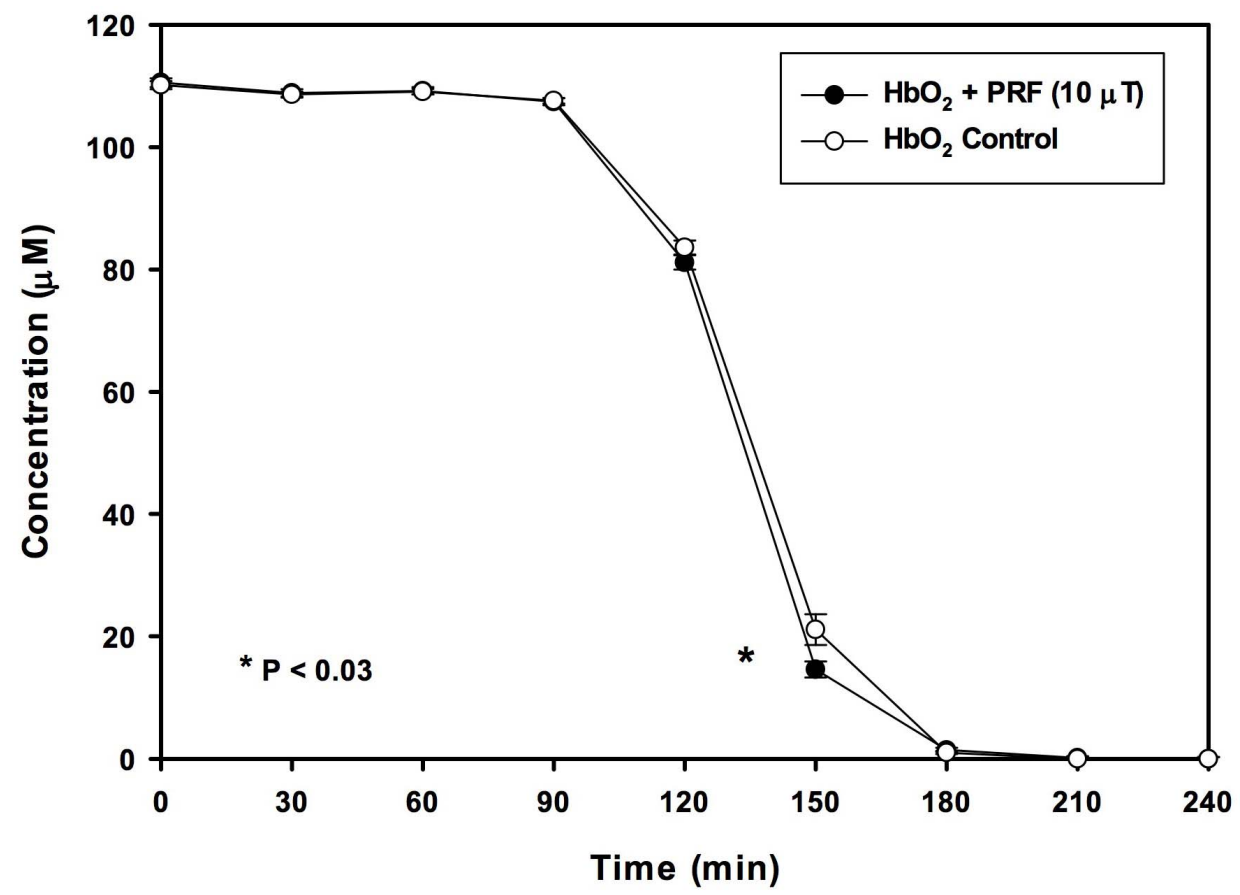

Figure 2. Effect of the pulsed radiofrequency field on time course of hemoglobin deoxygenation. Time course of oxy hemoglobin $\left(\mathrm{HbO}_{2}\right)$ concentration, after a single $30 \mathrm{~min}$ pulsed radiofrequency electromagnetic field (PRF) exposure of $\mathrm{Hb}$, under deoxygenating conditions. Concentration was determined by visible light spectroscopy at 560,576, $630 \mathrm{~nm}$. PRF exposure resulted in a significant (30.8 \pm 10.3$) \%$ reduction in oxyHb concentration, as compared to controls $(14.6 \pm 1.3 \mu \mathrm{M}$ vs. $21.1 \pm 2.5 \mu \mathrm{M}, \mathrm{P}<0.03, \mathrm{n}=5)$, suggesting an alteration in Hb solution properties that persisted after PRF signal was removed.

doi:10.1371/journal.pone.0061752.g002 


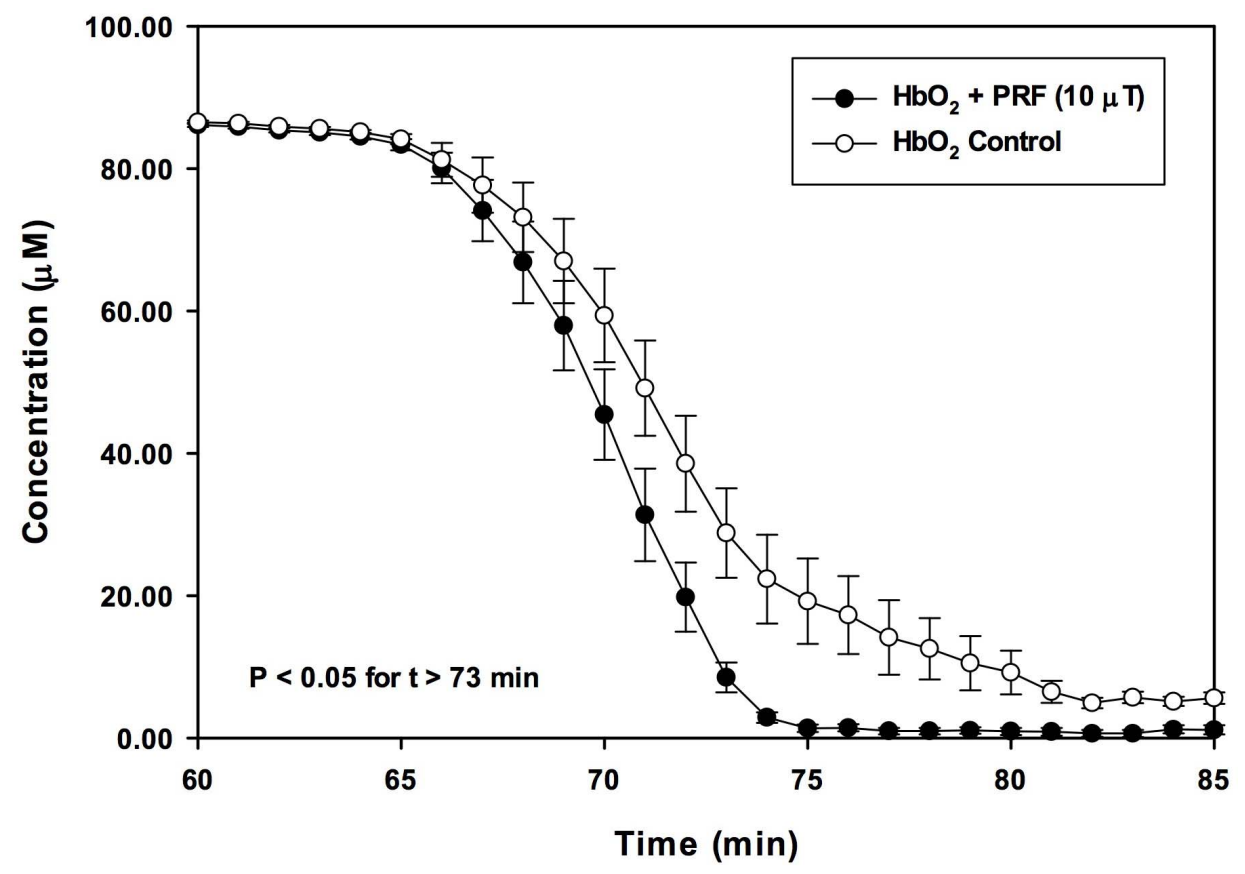

Figure 3. Addition of $\mathbf{5} \mathbf{M}$ urea to reaction mixture. Addition of $5 \mathrm{M}$ urea to the deoxygenation assay reduces the time required for deoxygenation and render the PRF effect more apparent. The maximal difference between PRF-treated and control samples occurred at 73 min, with PRF-treated samples showing a significant $(70.4 \pm 9.7) \%$ reduction in oxyHb concentration, as compared to controls $(8.5 \pm 2.1 \mu \mathrm{M}$ vs. $28.8 \pm 6.3 \mu \mathrm{M}$, $\mathrm{P}<0.03, \mathrm{n}=5$ ), in contrast to the $30.8 \%$ reduction observed in the absence of urea (cf. Figure 2 ). doi:10.1371/journal.pone.0061752.g003

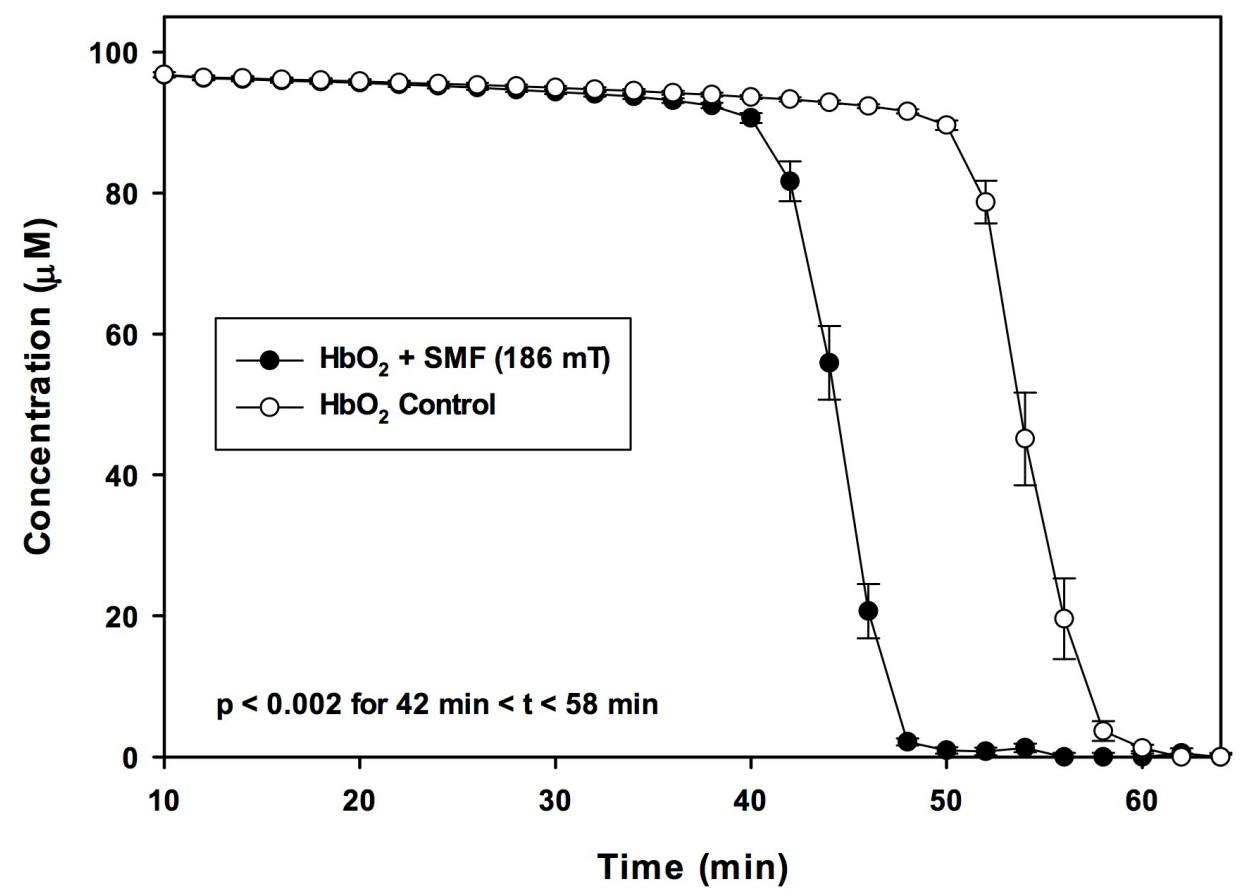

Figure 4. Effect of the static magnetic field on time course of hemoglobin deoxygenation. Hb deoxygenation after 10 min exposure to $186 \mathrm{mT}$ static magnetic field (SMF) in 5M urea. No significant change in oxy/deoxy ratio was visible until the time of rapid deoxygenation, at approximately $40 \mathrm{~min}$. The time of most rapid deoxygenation occurred approximately 10 min earlier for SMF treated samples $(\mathrm{P}<0.002$ for $42 \min <t<58 \min , \mathrm{n}=5$ ).

doi:10.1371/journal.pone.0061752.g004 


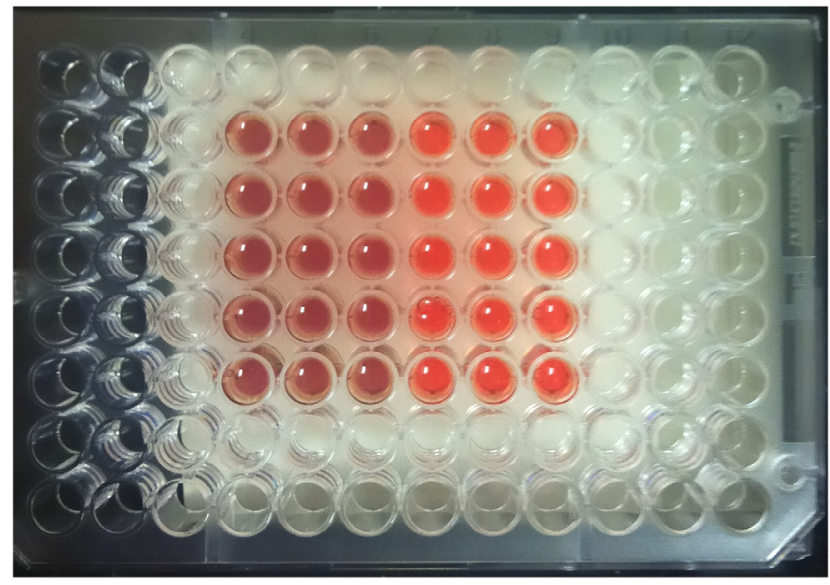

Figure 5. Reaction plate showing that addition of 5M urea to reaction solution renders magnetic field effect more apparent. 96-well spectrophotometer plate shown at 46 min after initiation of reaction of hemoglobin with dithiothreitol (DTT). Samples treated for $10 \mathrm{~min}$ with $186 \mathrm{mT}$ static magnetic field (3 left-hand columns in plate) substantially completed (see Figure 4) DTT-induced deoxygenation before untreated samples began to lose $\mathrm{O}_{2}$, rendering the magnetic field effect visibly apparent.

doi:10.1371/journal.pone.0061752.g005

suggests that more than one mechanism may be responsible for the results reported here. Although direct action on ferrous heme [28] or free radical lifetimes $[29,30]$ may account for the SMF effects observed here, several other models have been proposed for weak, $\mu$ T-range magnetic field bioeffects [31]. We have previously shown using the Lorentz-Langevin model that $\mu \mathrm{T}$-range magnetic fields may have an effect on the thermally activated orientation of ionic oscillators and waters bound at the protein surface $[32,33]$ and that the minimum magnetic field required to directly compete with thermal forces to affect dissociation of a bound ion or ligand from a protein binding site is in the 1-10 mT-range [34]. Thus, the effect of the mT-range magnetic field employed in this study could occur by the direct action of the Lorentz force on charges

\section{References}

1. Pilla AA (2006) Mechanisms and therapeutic applications of time varying and static magnetic fields. In: Barnes F, Greenebaum B, editors. Biological and Medical Aspects of Electromagnetic Fields. Boca Raton, CRC Press. pp. 351411 .

2. Pilla A, Fitzsimmons R, Muehsam D, Wu J, Rohde C, et al. (2011) Electromagnetic fields as first messenger in biological signaling: Application to calmodulin-dependent signaling in tissue repair. Biochim Biophys Acta 1810: 1236-1245.

3. Pilla AA (2012) Electromagnetic fields instantaneously modulate nitric oxide signaling in challenged biological systems. Biochem Biophys Res Commun 426 : 330-333.

4. Diniz P, Soejima K, Ito G (2002) Nitric oxide mediates the effects of pulsed electromagnetic field stimulation on the osteoblast proliferation and differentiation. Nitric Oxide. 7: 18-23.

5. Cheng G, Zhai Y, Chen K, Zhou J, Han G, et al. (2011) Sinusoidal electromagnetic field stimulates rat osteoblast differentiation and maturation via activation of NO-cGMP-PKG pathway. Nitric Oxide. 25: 316-325.

6. Fitzsimmons RJ, Gordon SL, Kronberg J, Ganey T, Pilla AA (2008) A pulsing electric field (PEF) increases human chondrocyte proliferation through a transduction pathway involving nitric oxide signaling. J Orthop Res. 26: 854 859.

7. Tao R, Huang K (2011) Reducing blood viscosity with magnetic fields. Phys Rev E Stat Nonlin Soft Matter Phys 84(1): 011905.

8. Sosa M, Bernal-Alvarado J, Jiménez-Moreno M, Hernández JC, GutiérrezJuárez G, et al. (2005) Magnetic field influence on electrical properties of human blood measured by impedance spectroscopy. Bioelectromagnetics 26: 564-570.

9. Shalaby TE, Shawki MM (2006) Biophysical and biochemical changes in the characteristics of rat blood exposed to combined alternating and static magnetic fields. Rom. J. Biophys 16: 169-180. bound at the protein/water interface. Functionally important hemoprotein molecular motions are slaved to the thermal fluctuations of the bulk solvent [35] and protein hydration plays a fundamental role in the stability of dynamics between $\mathrm{Hb}$ T-R conformations [36] so that the PRF and SMF interactions described above, acting at the protein/water interface, may suggest a means by which EMF could modulate protein function.

The deoxygenating conditions employed here, using the reducing agent DTT in an in vitro cell-free model, differ substantially from those found in vivo. However, EMF-induced changes in the structure and function of $\mathrm{Hb}$ and erythrocyte suspensions shown by others [7-12,14] have been reported in vivo and in aqueous solution (i.e. without chemical deoxygenating agents), thus demonstrating an EMF sensitivity under physiological conditions and in the absence of reducing agents. Enhanced delivery of oxygen has been shown to reduce inflammation [37] and enhance tissue repair [38] and at least one trial has reported an EMF-induced increase in deoxyHb in an in vivo animal model [12]. Allosteric modification of $\mathrm{Hb}$ has been suggested as a clinically useful means of enhancing oxygen delivery [39], and is in development for in vivo treatment of ischemia from stroke, cardiac disease and diabetic ulcers [15]. Although much further work is required to ascertain the clinical relevance of the results reported here, enhanced oxygen delivery using PRF or SMF may be important non-invasive, non-pharmacologic therapeutic modalities by which clinically relevant EMF-mediated enhancement of growth and repair processes can occur.

\section{Acknowledgements}

We would like to thank Dr. Joel Friedman from Albert Einstein College of Medicine, for suggesting the use of urea to enhance the EMF sensitivity.

\section{Author Contributions}

Conceived and designed the experiments: DM RL DC PL. Performed the experiments: DM. Analyzed the data: DM RL DC. Contributed reagents/ materials/analysis tools: RL DC PL PA AB MM. Wrote the paper: DM PL RL PA AB MM FB GA AP DC.

10. Sakhnini L, Khuzaie R (2001) Magnetic behavior of human erythrocytes at different hemoglobin states. Eur Biophys J. 30: 467-470.

11. Iwasaka M, Miyakoshi J, Ueno S (2001) Optical absorbance of hemoglobin and red blood cell suspensions under magnetic fields. IEEE Trans Magnetics 37: 2906-2908.

12. Milweski S, Szczepański W (2006) Effects of electromagnetic fields on the meat performance and wool performance of sheep Arch. Tierz., Dummerstorf 49(Special Issue): 219-225.

13. Mousavy SJ, Riazi GH, Kamarei M, Aliakbarian H, Sattarahmady N, et al. (2009) Effects of mobile phone radiofrequency on the structure and function of the normal human hemoglobin. Int J Bio Macromolecules 44: 278-285.

14. Magazù S, Calabrò E, Campo S (2010) FTIR spectroscopy studies on the bioprotective effectiveness of trehalose on human hemoglobin aqueous solutions under $50 \mathrm{~Hz}$ electromagnetic field exposure. J Phys Chem. 114: 12144-12149.

15. Najjar SS, Bottomley PA, Schulman SP, Waldron MM, Steffen RP, et al. (2005) Effects of a pharmacologically-induced shift of hemoglobin-oxygen dissociation on myocardial energetics during ischemia in patients with coronary artery disease. J Cardiovasc Magn Reson 7: 657-666.

16. Herzfeld J, Seidel NE, Taylor MP, Droupadi PR, Wang NE (1990) Gentle chemical deoxygenation of hemoglobin solutions. Hemoglobin 14: 399-411.

17. Benesch RE, Benesch R, Yung S (1973) Equations for the spectrophotometric analysis of hemoglobin mixtures. Anal Biochem 55: 245-248.

18. Pilla AA, Muehsam DJ, Markov MS, Sisken BF (1999) EMF signals and ion/ ligand binding kinetics: prediction of bioeffective waveform parameters. Bioelectrochem Bioenerg. 48: 27-34.

19. Strauch B, Patel MK, Navarro A, Berdischevsky M, Pilla AA (2007) Pulsed magnetic fields accelerate wound repair in a cutaneous wound model in the rat. Plast Reconstr Surg 120: 425-430. 
20. Strauch B, Patel MK, Rosen DJ, Mahadevia S, Brindzei N, et al. (2006) Pulsed magnetic field therapy increases tensile strength in a rat Achilles' tendon repair model. J Hand Surg. 31. 1131-1135.

21. RasouliJ, Lekhraj R, White NM, Flamm ES, Pilla AA, et al. (2012) Attenuation of interleukin-1beta by pulsed electromagnetic fields after traumatic brain injury. Neurosci Lett. 519: 4-8.

22. Hedén P, Pilla AA (2008) Effects of pulsed electromagnetic fields on postoperative pain: a double-blind randomized pilot study in breast augmentation patients. Aesthetic Plast Surg 32: 660-666.

23. Rohde C, Chiang A, Adipojou O, Casper D, Pilla A (2010) Effects of Pulsed Electromagnetic Fields on IL-1 $\beta$ and Post Operative Pain: A Double-Blind, Placebo-Controlled Pilot Study in Breast Reduction Patients. Plast Reconstr Surg. 125: 1620-1629.

24. Nelson FR, Zvirbulis R, Pilla AA (2012) Non-invasive electromagnetic field therapy produces rapid and substantial pain reduction in early knee osteoarthritis: a randomized double-blind pilot study. Rheumatol Int Mar 27. [Epub ahead of print]

25. Eccles NK (2005) A critical review of randomized controlled trials of static magnets for pain relief. J Altern Complement Med 11(3): 495-509.

26. Roche CJ, Guo F, Friedman JM (2006) Molecular level probing of preferential hydration and its modulation by osmolytes through the use of pyranine complexed to hemoglobin. J Biol Chem 281: 38757-38768.

27. Royer WE Jr, Pardanani A, Gibson QH, Peterson ES, Friedman JM (1996) Ordered water molecules as key allosteric mediators in a cooperative dimeric hemoglobin. Proc Natl Acad Sci U S A. 93(25): 14526-31.

28. Pauling L (1977) Magnetic properties and structure of oxyhemoglobin. Proc Natl Acad Sci USA. 74: 2612-2613.
29. Closs GL (1969) Mechanism explaining nuclear spin polarizations in radical combination reactions. J Am Chem Soc 91: 4552-4554.

30. Timmel CR, Till U, Brocklehurst B, McLauchlan KA, Hore PJ (1998) Effects of weak magnetic fields on free radical recombination reactions. Molecular Physics 95: 71-89.

31. Binhi VN, Rubin AB (2007) Magnetobiology: the kT paradox and possible solutions. Electromagn Biol Med 26: 45-62.

32. Muehsam DJ, Pilla AA (2009) A Lorentz model for weak magnetic field bioeffects: part I -thermal noise is an essential component of AC/DC effects on bound ion trajectory. Bioelectromagnetics 30: 462-475.

33. Muehsam DJ, Pilla AA (2009) A Lorentz model for weak magnetic field bioeffects: part II - secondary transduction mechanisms and measures of reactivity. Bioelectromagnetics 30: 476-488.

34. Muehsam DJ, Pilla AA (1996) Lorentz approach to static magnetic field effects on bound ion dynamics and binding kinetics: thermal noise considerations. Bioelectromagnetics 17: 89-99.

35. Frauenfelder H, Chen G, Berendzen J, Fenimore PW, Jansson H, et al. (2009) A unified model of protein dynamics. Proc Natl Acad Sci USA 106(13):5129-34.

36. Salvay AG, Grigera JR, Colombo MF (2003) The role of hydration on the mechanism of allosteric regulation: in situ measurements of the oxygen-linked kinetics of water binding to hemoglobin. Biophys J 84(1): 564-70.

37. Bitterman H (2007) Oxygen: an anti-inflammatory drug. Isr Med Assoc J 9: $874-876$.

38. Tandara AA, Mustoe TA (2004) Oxygen in wound healing-more than a nutrient. World J Surg. 28: 294-300.

39. Papassotiriou I, Kister J, Griffon N, Stamoulakatou A, Abraham DJ (1998) Modulating the oxygen affinity of human fetal haemoglobin with synthetic allosteric modulators. Br J Haematol. 102: 1165-1171. 